OPEN ACCESS

Edited by:

Dipak Panigrahy,

Harvard Medical School,

United States

Reviewed by:

Stefania Ceruti,

University of Milan, Italy

Shun-Chin Yang,

Taipei Veterans General Hospital,

Taiwan

*Correspondence:

Alessandro Leut

a.leuti@unicampus.it

Mauro Maccarrone

mauro.maccarrone@univaq.it

${ }^{\dagger}$ These authors share first authorship

Specialty section:

This article was submitted to Inflammation Pharmacology,

a section of the journal

Frontiers in Pharmacology

Received: 31 May 2021

Accepted: 27 July 2021

Published: 11 August 2021

Citation:

Leuti A, Fava M, Pellegrini $N$ and

Maccarrone M (2021) Role of Specialized Pro-Resolving Mediators in Neuropathic Pain.

Front. Pharmacol. 12:717993. doi: 10.3389/fphar.2021.717993

\section{Role of Specialized Pro-Resolving Mediators in Neuropathic Pain}

\author{
Alessandro Leuti ${ }^{1,2 * t}$, Marina Fava ${ }^{2,3 \dagger}$, Niccolò Pellegrini ${ }^{1}$ and Mauro Maccarrone ${ }^{2,4 *}$ \\ ${ }^{1}$ Department of Medicine, Campus Bio-Medico University of Rome, Rome, Italy, ${ }^{2}$ European Center for Brain Research/IRCCS \\ Santa Lucia Foundation, Rome, Italy, ${ }^{3}$ Faculty of Biosciences and Technology for Food Agriculture and Environment, University of \\ Teramo, Teramo, Italy, ${ }^{4}$ Department of Biotechnological and Applied Clinical Sciences, University of L'Aquila, L'Aquila, Italy
}

Inflammation and neuroinflammation are critical mechanisms in the generation of neuropathic pain that is experienced in several chronic diseases. The aberrant inflammation that triggers this pathophysiologic process can be tracked down to an exacerbated immune response, which establishes a vicious cycle and continuously recruits inflammatory cells by inducing chronic tissue damage. Recently, impairment of the cellular and molecular machinery orchestrated by specialized pro-resolving mediators (SPMs) - i.e., endogenous lipids termed resolvins, protectins, maresins, and lipoxins that confine the inflammatory cascades in space and time during the "resolution of inflammation"-has emerged as a crucial event in the derangement of the inflammatory homeostasis and the onset of chronic inflammation and pain. Indeed, a deviant inflammatory response that is not adequately controlled by the resolution network leads to the overproduction of pro-inflammatory eicosanoids that, opposite to SPMs, lead to neuropathic pain. Interestingly, in the last two decades convincing evidence has demonstrated that SPMs antagonize the in vivo activity of pro-inflammatory eicosanoids and, overall, exert potent anti-hyperalgesic effects in a number of pain-associated paradigms of disease, such as arthritis and chemotherapy-induced peripheral neuropathy, as well as in many experimental models of pain like mechanical allodynia, chemical pain, heat hypersensitivity and phase 1 and 2 inflammatory pain. Of note, accumulated evidence supports a synergy between SPMs and other signalling pathways, such as those mediated by transient receptor potential (TRP) channels and those triggered by opioid receptors, suggesting that the cascade of events where inflammation and pain perception take part might be ways more intricated than originally expected. Here, we aim at presenting a state-of-the-art view of SPMs, their metabolism and signalling, in the context of cellular and molecular pathways associated to neuropathic pain.

Keywords: resolution of inflammation, chronic inflammation, neuropathic pain, neuroinflammation, resolution of inflammatory pain

\section{INTRODUCTION}

Pain and chronic neuropathic pain represent a significant social issue, which in Europe affects approximately $20 \%$ of the general population (Breivik et al., 2006). This condition can manifest as spontaneous pain, mechanical allodynia-i.e., pain caused by mechanical non-painful stimuli-or hyperalgesia-i.e., increased sensibility to pain (Baron, 2000; Baron, 2006). In general, neuropathic 
pain arises from a damage of the central or peripheral nervous system occurring during different pathologies, with the sources of this alteration being vastly heterogeneous. Indeed, peripheral neuropathies can arise from a number of localized lesions of the peripheral nerves, from peripheral polyneuropathies (as in diabetes mellitus) or from central lesions that might occur during multiple sclerosis (MS) or spinal cord injury (SCI), as reviewed in Baron (2006). Also enhanced sensitivity of peripheral somatosensory routes is a frequent cause of exacerbated pain, and is elicited by the presence of pro-inflammatory molecules like prostaglandins that are synthesized during acute and chronic inflammation (Jang et al., 2020), as well as by the aberrant activation of the immune response. The pathophysiological elements that trigger this deviant inflammatory response are not yet completely understood, though they have been often linked to persistent inflammation and neuroinflammation (Leuti et al., 2020), which act as a crucial element of neuropathic and inflammatory pain (Kidd and Urban, 2001; Baron, 2006). Inflammation represents a defensive cellular and molecular network, which evolved to avoid the invasion of microbial pathogens that might compromise tissue homeostasis. This process is designed-at least in principle-as a self-resolving mechanism, which is supposed to be confined in space, time and magnitude to avoid tissue damage that abnormal production of pro-inflammatory mediators (e.g., cytokines, eicosanoids) and immune activation would inevitably cause. However, deranged inflammation can lead to a sustained immune response that acts in a vicious cycle, leading to tissue damage, fibrosis, loss of tissue function, and notably to persistent pain by both triggering chronic damage to neural tissues and producing inflammatory mediators that trigger peripheral hypersensitization.

Transition from acute to chronic inflammation has recently emerged as the result of impairment or lack of efficiency of a process termed "resolution of inflammation", during which a novel class of bioactive lipids called "specialized pro-resolving mediators" (SPMs) would normally coordinate the regression of the immune response by antagonizing both the production and the action of inflammatory mediators that continuously fuel aberrant phlogosis (Serhan and Levy, 2018). Consequently, inefficient resolution caused by insufficient production of SPMs, or lack of response to their homeostatic action, has been shown in a number of independent studies to be associated to chronic pathologies (Leuti et al., 2020). In this context, SPMs work as potent anti-inflammatory/pro-resolving agents which are increasingly emerging as promising targets in the quest for pharmacological strategies able to counteract neuropathic pain during neuroinflammatory diseases.

\section{SPECIALIZED PRO-RESOLVING MEDIATORS AND THEIR METABOLISM}

All SPMs are produced from essential polyunsaturated fatty acids (PUFAs) like arachidonic, docosahexaenoic and eicosapentaenoic acid (AA, DHA, and EPA, respectively), that are introduced in the organism through the diet. These are esterified to the membrane phospholipids, before being released through the action of phospholipase $\mathrm{A}_{2}\left(\mathrm{PLA}_{2}\right)$. These precursors are converted into the final products by the concerted action of different lipoxygenase (LOX) isozymes, namely 5-, 12and 15-LOX, expressed by granulocytes, macrophages, platelets, and endothelial cells. The synthesis of these compounds is triggered by acute inflammation, so that they can antagonize the phlogistic signals by counteracting granulocyte chemotaxis and activation of both innate and adaptive immune cells. To this aim AA, DHA and EPA derivatives trigger differentiation of tolerogenic macrophage phenotypes, by inducing clearance of dead cells and tissue debris and, overall, orchestrating tissue regeneration, and return to normal tissue homeostasis (Chiurchiù et al., 2016; Serhan and Levy, 2018; Leuti et al., 2020). The main metabolic pathways that lead to the synthesis of SPMs, along with their known target receptors, are illustrated in Figure 1.

\section{AA-Derived SPMs}

Lipoxin $\mathrm{A}_{4}\left(\mathrm{LXA}_{4}\right)$ and $\mathrm{LXB}_{4}$ are the only two known members of this group, and are synthesized by the concerted action of granulocytes, platelets, endothelial cells, and macrophages from phospholipid-derived AA. Their production involves two biosynthetic pathways: the first is in common with that of leukotrienes, because leukotriene $\mathrm{A}_{4}\left(\mathrm{LTA}_{4}\right)$ is converted into $\mathrm{LXA}_{4}$ and $\mathrm{LXB}_{4}$ in a 12-LOX-dependent manner (Serhan et al., 1986). The second route involves 15-LOX-dependent hydroperoxidation of AA, and its 5-LOX-driven conversion into an epoxide, which is eventually hydrolyzed to yield the final LXs (Romano et al., 2015). Acetylation of cyclooxygenase- 2 by aspirine (aa-COX-2) gives rise to a series of LX epimers, called aspirin-triggered (AT) lipoxins AT-LXA and AT-LXB 4 (Clària and Serhan, 1995). Formyl peptide receptor 2 (FPR2), also known as ALX, and GPR32 mediate the effects of $\mathrm{LXA}_{4}$ and AT-LXA , and remain as yet the only receptors known to bind LXs (Chiang and Serhan, 2017).

\section{DHA-Derived SPMs}

DHA-derived SPMs represent the largest group of SPMs, and include three main classes: $\mathrm{D}$-series resolvins $1-6\left(\mathrm{RvD}_{1-6}\right)$, maresins 1 and $2\left(\mathrm{MaR}_{1}\right.$ and $\left.\mathrm{MaR}_{2}\right)$, and (neuro)protectins 1 and $\mathrm{X}\left(\mathrm{PD}_{1}\right.$ and $\left.\mathrm{PD}_{\mathrm{X}}\right)$. These SPMs are produced by LOXcatalyzed differential insertion of oxygen in the carbon backbone of DHA, to yield one of two hydroperoxyls (Hp): i) 15-LOX yields a $17(S)$-hydroperoxyl derivative (17(S)-HpDHA), which is then converted into different RvDs or into $\mathrm{PD}_{1}$ by 5 LOX; ii) alternatively, 12-LOX yields $14(S)$-HpDHA, which can be converted into its corresponding $13(S)-14(S)$ epoxide, and then is hydrolyzed into the MaR series. Interestingly, DHA can also be converted into 17(R)-HpDHA by aa-COX2, and the latter compound is converted by 5-LOX into a group of epimeric substances called aspirin-triggered (AT)-RvDs (Serhan and Levy, 2018).

Despite the considerable number of molecules that have been characterized in this group-which also includes the corresponding cysteinyl derivatives of Rvs, protectins and MaRs-only a handful of receptors have been discovered so far: RvD1, RvD3, and RvD5 engage GPR32, while RvD1 is 

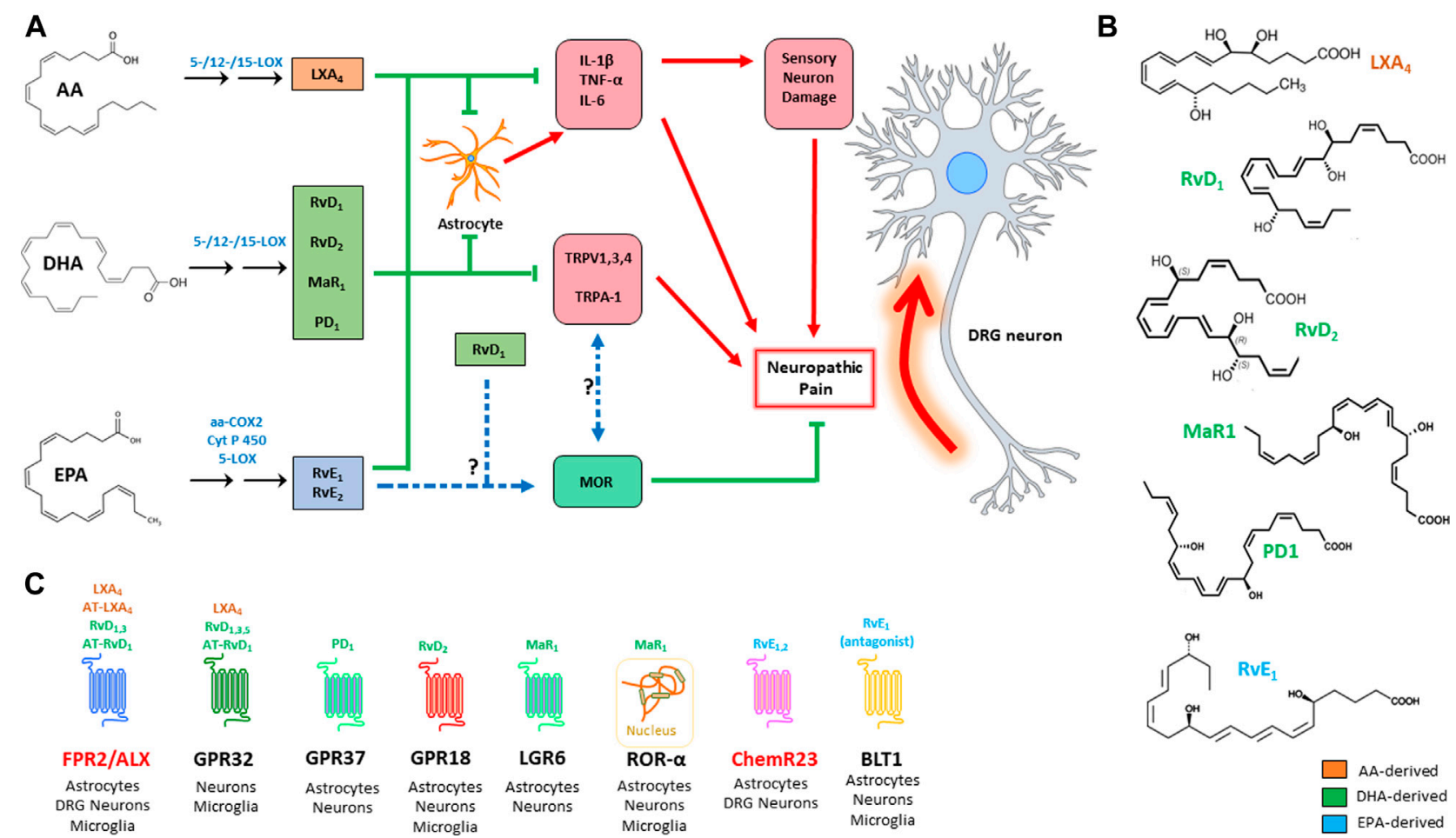

C
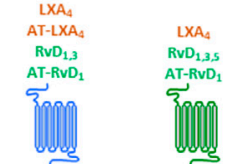

FPR2/ALX

Astrocytes

RG Neurons

Neurons

Microglia
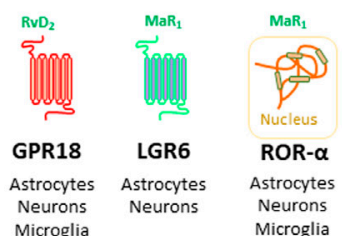

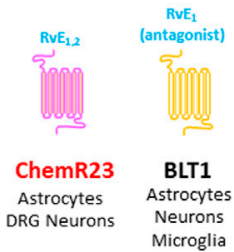

FIGURE 1 | Action of the main SPMs on neuropathic pain (A): SPMs modulate the genesis of neuropathic pain by acting on three main targets: i) inhibition of proinflammatory signals; ii) inhibition of TRP channels; and iii) modulation of opioid signalling. Chemical structure of the main SPMs involved in neuropathic pain (B). SPM receptors, with their respective ligands and distribution; receptors that are highlighted in red are those that have been more directly investigated in pain studies (C). AA: arachidonic acid; aa-COX-2: aspirin-acetylated cyclooxygenase 2; ALX/FPR2: formyl peptide receptor 2; AT: aspirin triggered; BLT1: leukotriene B4 receptor 1; ChemR23: chemerin receptor 23; COX: cyclooxygenase; CYP450: cytochrome P450; DHA: docosahexaenoic acid; EPA: eicosapentaenoic acid; GPR: G-proteincoupled receptor; IL: interleukin; LGR6: leucine-rich containin G-protein coupled receptor 6; LOX: lipoxygenase; LX: lipoxin; MaR: maresin; MOR: $\mu$-opioid receptor; PD: protectin; Rv: resolvin; TNF: tumor necrosis factor; TRPV1: transient receptor potential cation channel subfamily V member 1; TRPA: transient receptor potential cation channel subfamily A; ROR: RAR-related orphan receptor.

also able to bind (much alike $\mathrm{LXA}_{4}$ ) FPR2/ALX (Figure 1C). In addition, $\mathrm{RvD}_{2}$ engages GPR18, while $\mathrm{PD}_{1}$ and $\mathrm{MaR}_{1}$ have only recently been characterized as agonists of GPR37 and LGR6, respectively (Serhan and Levy, 2018). Of note, $\mathrm{MaR}_{1}$ is also able to bind the intracellular retinoic acid-related orphan receptor $a$ (RORa), as recently reported (Han et al., 2019).

\section{EPA-Derived SPMs}

EPA derived E-series resolvins (i.e., $\mathrm{RvE}_{1-3}$ ) are synthesized in a rather peculiar way, if compared to the other SPMs, because the synthesis of their main common precursor, $18(R)$-HpEPE, is catalyzed by aa-COX2 in vascular endothelial cells, or alternatively by cytochrome P450 (CYP450). Then, 18 (R)$\mathrm{HpEPE}$ can be converted by $12-\mathrm{LOX}$ and $15-\mathrm{LOX}$ into $\mathrm{RvE}_{3}$, while 5-LOX converts it into $\mathrm{RvE}_{1}$ and $\mathrm{RvE}_{2}$. E-series resolvins are rather unique in the way they engage their target receptors during resolution of inflammation: in particular, $\mathrm{RvE} 1$ is the only known pro-resolving lipid that acts both as an agonist to its own receptor-i.e., Chemerin Receptor 23 (ChemR23) - and as an antagonist to the leukotriene $\mathrm{B}_{4}$ receptor 1 (BLT1) (Serhan and Levy, 2018). Instead, all the other SPMs seem to exclusively activate pro-resolving receptors, often being able to act on different targets. Finally, $\mathrm{RvE}_{2}$ acts as a partial agonist to ChemR23 (Oh et al., 2012).

\section{SPMs IN PAIN}

The antinociceptive activity of SPMs has been mostly correlated to their ability to counteract the inflammatory surge that is unleashed during inflammatory response, which in turn causes both hyperactivation of pain neural routes and inflammatory damage leading to chronic neuropathic pain. However, in the past 2 decades SPMs have been consistently proven to act on pain perception via a cross-talk with other signal transduction systems that directly control nociception at the neural level, such as those driven by opioid receptors and transient receptor potential (TRP) channels. In particular, TRP receptors are cation-permeable membrane channels which are gated by physical (e.g., hot or cold) or chemical (e.g., endogenous lipids, or molecules found in foods and spices) stimuli; upon their activation, they drive the depolarization of the dorsal root ganglia (DRG) sensitive neurons by causing $\mathrm{Ca}^{2+}$ and $\mathrm{Na}^{+}$influx (Jardín et al., 2017). Relevant to this work, SPMs represent the first endogenous inhibitors of 
TABLE 1 | Main effects of AA-, DHA-, and EPA-derived SPMs on neuropathic pain. AA: arachidonic acid; aa-COX-2: aspirin-acetylated cyclooxygenase 2; ALX/FPR2: formyl peptide receptor 2; AT: aspirin triggered; BDNF: brain-derived neurotrophic factor; BLT1: leukotriene B4 receptor 1; CFA: complete Freund's adjuvant; ChemR23: chemerin receptor 23; COX: cyclooxygenase; CYP450: cytochrome P450; DHA: docosahexaenoic acid; DM: diabetes mellitus; DRG: dorsal root ganglia; EPA: eicosapentaenoic acid; EPSC: excitatory postsynaptic current; GPR: G-protein-coupled receptor; LGR6: leucine-rich containin G-protein coupled receptor 6; IL: interleukin; LOX: lipoxygenase; LX: lipoxin; MIA: monoiodoacetate; MaR: maresin; MOR: $\mu$-opioid receptor; NDMA: N-methyl D-aspartate; OA: osteoarthritis; PD: protectin; Rv: resolvin; SCI: spinal cord injury; TRPV1: transient receptor potential cation channel subfamily V member 1; TNF: tumor necrosis factor; TRPA: transient receptor potential cation channel subfamily A; ROR: RAR-related orphan receptor.

\begin{tabular}{|c|c|c|c|}
\hline $\begin{array}{l}\text { AA-derived } \\
\text { SPMs }\end{array}$ & Effects & Disease model & Reference \\
\hline \multirow[t]{8}{*}{$L X A_{4} / A T-L X$} & Reduces inflammatory hyperalgesia & $\begin{array}{l}\text { Formalin-/carrageenan-/CFA-induced } \\
\text { pain }\end{array}$ & Svensson et al. (2007) \\
\hline & Reverts morphine tolerance & Tail flick test & Tian et al. (2015) \\
\hline & Alleviates mechanical allodynia & Neuropathic pain & Wang et al. (2014) \\
\hline & Inhibits NF-kB & $\begin{array}{l}\text { Hernia, DRG compressive pain, } \\
\text { carrageenan-induced pain }\end{array}$ & $\begin{array}{l}\text { Svensson et al. (2007); Sun et al. (2012); } \\
\text { Miao et al. (2015) }\end{array}$ \\
\hline & Reduces TNF- $\alpha, I L-1 \beta$, and IL-6 production & Carragenaan-treated mice & $\begin{array}{l}\text { Abdelmoaty et al. (2013); Hu et al. (2012); } \\
\text { Wang et al. (2014) }\end{array}$ \\
\hline & Counteracts nociceptin action & Mouse air pouch & Serhan et al. (2001) \\
\hline & Interferes with IL-1 $\beta$ maturation & Neuropathic pain & Li et al. (2013) \\
\hline & Inhibits activation of spinal microglia & $\mathrm{SCl}$ rat model & Martini et al. (2016) \\
\hline
\end{tabular}

\begin{tabular}{ll}
$\begin{array}{l}\text { DHA-derived } \\
\text { SPMs }\end{array}$ & Effects \\
\hline AT-RvD $_{1}$ & $\begin{array}{l}\text { Reduces weight-bearing asymmetry and hind paw withdrawal } \\
\text { Abates the production of pro-inflammatory factors e.g. TNF- } \alpha \text { and } \\
\text { IL-1 } \beta\end{array}$ \\
& $\begin{array}{l}\text { Exerts anti-neuroinflammatory action mediated by downregulation } \\
\text { of 5-LOX activating protein (FLAP) }\end{array}$ \\
& Is associated with pain perception
\end{tabular}

Disease model

Reference

$17(R)-\mathrm{HDHA} \quad$ Is associated with pain perception

Blunts astrogliosis in the spinal cord

MIA

Arthritis

Carragenaan-treated mice

$\mathrm{OA}$

OA

OA/sciatica

cord and in the neurons of DRG

ALX (receptor) Spinal overexpression

$\mathrm{RvD}_{1}$

$\mathrm{PD}_{1}$

Drives PPAR $\gamma$-dependent analgesic effects

Improves tissue regeneration due to M2 macrophages

Reduces neuroinflammation and phosphorylation of NMDA

receptors in the neurons of the thoracic spinal dorsal horn

Prevents heat-induced pain, inflammatory hypersensitivity and nociception-related EPSC

Reduces mechanical allodynia and heat hyperalgesia

Analgesic

Prevents heat-induced pain behaviour, inflammatory

hypersensitivity and nociception-related EPSC

Reduces glial reaction in mouse models

Inhibits TRPV1 in DRG-derived neurons

Reduces mechanical allodynia

Induces the expression of BDNF

GPR37

(receptor)

$\mathrm{MaR}_{1}$
Blunts inflammation, heat hyperalgesia and mechanical allodynia

Inhibits the activation of DRG neurons and abates the activation of spinal NF-kB

Reduces mechanical hypersensitivity due to inhibition of macrophage inflammatory chemotaxis in the DRG

\section{Carrageenan-induced pain}

DM

DM

Chronic pancreatitis

Sepsis

Bone tumour

Post-operative neuropathic pain

Sepsis

Sciatic nerve transection

Chemically/pharmacologically-induced

pain

Sciatic nerve axotomy and spinal nerve ligation

Ocular pathology

$\mathrm{GPR}^{-1-}$ mice

Carrageenan-induced and radicular pain, spinal nerve ligation

Arthritic pain
Huang et al. (2017)

Lima-Garcia et al. (2011)

Meesawatsom et al. (2016)

Valdes et al. (2017)

Huang et al. (2017)

Lima-Garcia et al. (2011); Liu et al. (2016)

Meesawatsom et al. (2016); Huang et al. (2017)

Saito et al. (2015)

Saito et al. (2015)

Quan-Xin et al. (2012)

Bang et al. (2010); Park et al. (2011b)

Khasabova et al. (2020)

Huang et al. (2011); Wang and Strichartz, (2017); Zhang et al. (2018)

Bang et al. (2010); Park et al. (2011b)

Xu et al. (2013)

Park et al. (2011a)

Xu et al. (2013)

Pham et al. (2017); Pham and Bazan, (2021)

Bang et al. (2018)

Fattori et al (2019); Gao et al. (2018); Wang et al. (2020)

Allen et al. (2020)

\begin{tabular}{ll}
$\begin{array}{l}\text { EPA-derived } \\
\text { SPMs }\end{array}$ & Effects \\
\hline $\mathrm{RVE}_{1}$ & Suppresses inflammatory spinal nociception \\
& $\begin{array}{l}\text { Modulates opioid signalling } \\
\text { Decreases bone cancer-associated pain } \\
\end{array}$ \\
& Inhibits TRPV1
\end{tabular}

Frontiers in Pharmacology | www.frontiersin.org pain

CFA-induced pain

Fibrosarcoma

Peripheral nerve injury
Formalin-/carrageenan-/CFA-induced Xu et al. (2010)
Oehler et al. (2017)

Khasabova et al. (2020)

Xu et al. (2010); Jo et al. (2016) 
TRPs documented so far (Park et al., 2011b; Lim et al., 2015). The main effects of AA-, DHA- and EPA-derived SPMs on neuropathic pain models are summarized in Table 1.

\section{AA-Derived SPMs}

Lipoxins (LXs) represent the first SPMs ever characterized, with the first reports describing their beneficial role in preventing neuropathic pain dating back to the first years of the $21^{\text {st }}$ century. These seminal works not only described the ability of $\mathrm{LXA}_{4}$, and of its aspirin-triggered epimer, to counteract the action of nociceptin and to directly reduce inflammatory hyperalgesia, but also documented for the first time the expression of FPR2/ ALX on spinal astrocytes (Serhan et al., 2001; Svensson et al., 2007).

Acute pro-inflammatory cytokines represent a primum movens in driving neuropathic pain, as well as a main target on which LXs act during the resolution of inflammatory pain. Indeed, intrathecal administration of $\mathrm{LXA}_{4}$ or AT-15-epi-LXA results in lower production of tumor necrosis factor (TNF)- $\alpha$ in carrageenan-induced spinal pain and in spinal astrocyte cultures (Abdelmoaty et al., 2013), in animal models of cancer-induced pain, where also interleukin (IL)- $1 \beta$ is modulated (Hu et al., 2012), as well as in SCI rat models, where they inhibit activation of spinal microglia (Martini et al., 2016).

Of note, a number of mechanistic studies have also investigated the intracellular pathways that mediate the therapeutic properties of LXs, unravelling a rather convoluted signalling network: AT-LXA 4 can hinder IL- $1 \beta$ maturation by directly targeting NLRP1 inflammasome, which results in reduced mechanical allodynia and thermal hyperalgesia in models of nerve ligation, as well as it reverts morphine tolerance in tail flick tests (Li et al., 2013; Tian et al., 2015). In a similar study, the same lipid alleviated mechanical allodynia caused by chronic constriction nerve injury by hindering JAK/ STAT signalling, thus reducing the production of TNF- $\alpha$, IL- $1 \beta$, and IL-6 (Wang et al., 2014). Further studies also clarified that cytokine modulation is exerted by LXs through different molecular mechanisms, in that intrathecal or systemic administration of $\mathrm{LXA}_{4}$ led to: i) inhibition of NF-kB, ii) disruption of JAK/STAT signalling-with subsequent downregulation of acute innate pro-inflammatory cytokines, and upregulation of anti-inflammatory cytokines such as IL-10 and TGF $\beta$-, iii) dephosphorylation of ERK and JNK signalling in non-compressive hernia, DRG compressive pain and carrageenan-induced models of chronic pain (Svensson et al., 2007; Sun et al., 2012; Miao et al., 2015).

Further evidence of the involvement of LX-related resolution of inflammation in neuropathic pain has been provided by a body of investigations-mostly performed during the first decade of the 2000s-reporting the antinociceptive effects of annexin 1, which was known as FPR2/ALX receptor before it was deorphanized as the $\mathrm{LXA}_{4}$ target (Chen et al., 2014).

\section{DHA-Derived SPMs}

DHA-derived SPMs have been thoroughly investigated in the context of pain research during the past 20 years. Treatment with $\mathrm{AT}-\mathrm{RvD}_{1}$, or its precursor 17(R)-HDHA, results in the enhancement of $\mathrm{RvD}_{2}$ production while consistently reducing weight-bearing asymmetry and hind paw withdrawal in monoiodoacetate (MIA)-treated mice (Huang et al., 2017). Moreover, it hinders complete Freund's adjuvant (CFA)induced arthritic pain in rats by abating the production of pro-inflammatory cytokines like TNF- $\alpha$ and IL-1 $\beta$ (LimaGarcia et al., 2011); interestingly, in the same studies, a lack of effect of SPM treatment on bona fide joint pathological features (such as joint oedema, chondropathy or synovitis) was reported, suggesting that the action on pain is the result of a direct activity on the inflammatory cues that drive nerve-mediated hyperalgic signals. Indeed, $17(R)$-HDHA was shown to blunt astrogliosis in the spinal cord (Huang et al., 2017), and to down-regulate NF-kB and COX2 in the dorsal horn of lumbar spinal cord and in DRG neurons (Lima-Garcia et al., 2011; Liu et al., 2016). Of note, circulating levels of $17(R)$-HDHA were also associated with pain perception in osteoarthritis patients (Valdes et al., 2017). In line with this, an independent study has recently demonstrated that the anti-neuroinflammatory action behind the analgesic properties of $\mathrm{AT}-\mathrm{RvD}_{1}$ was mediated by downregulation of the 5-LOX activating protein (FLAP) in the spinal cord of carrageenan-treated mice (Meesawatsom et al., 2016). Interestingly, pharmacological pain mouse models also displayed spinal overexpression of ALX and ChemR23, suggesting that the pro-resolving attempts of SPMs, though ultimately insufficient to confine phlogistic processes, might be exploited to treat neuropathic pain (Meesawatsom et al., 2016; Huang et al., 2017).

Remarkably, $\mathrm{RvD}_{1}$ and $\mathrm{RvD}_{2}$ have also been recently investigated with respect to their potential as analgesic agents in neuropathic post-operative pain, and their intrathecal administration has been shown to be beneficial in postthoracotomy, lateral paw incision, skin/muscle incision and retraction surgery (Huang et al., 2011; Wang and Strichartz, 2017; Zhang et al., 2018).

Several chronic pathologies display chronic pain as a debilitating feature that comes from peripheral neuropathy, and also SPMs have been investigated in a number of paradigms of the same disorders. For instance, $\mathrm{RvD}_{1}$ drives peroxisome proliferator-activated receptor- $\gamma$ (PPAR $\gamma$ )dependent analgesic effects in $d b / d b$ mice, that lack the leptin receptor (Saito et al., 2015). In this animal model of diabetes the effect of $\mathrm{RvD}_{1}$ was also associated to improved tissue regeneration via restoration of rosiglitazone-induced differentiation of M2 macrophages, which is impaired in $d b / d b$ mice (Saito et al., 2015). In addition, $\mathrm{RvD}_{1}$ counteracted chronic pancreatitisinduced mechanical allodynia in trinitrobenzene sulfonic acidtreated rats by reducing neuroinflammation and phosphorylation of $\mathrm{N}$-methyl D aspartate (NMDA) receptors in neurons of the thoracic spinal dorsal horn (Quan-Xin et al., 2012). Finally, RvD ${ }_{5}$ reduced mechanical allodynia in paclitaxel-treated mice, a wellknown pharmacological model of chemotherapy-induced peripheral neuropathy (Luo et al., 2019).

Interestingly, although the inhibition of the inflammatory/ neuroinflammatory cues that hyperactivate nociceptive routes is considered a main mechanism mediating analgesic properties of SPMs, accumulated evidence has also demonstrated a more direct 
action on nervous pain transmission, as well as an interaction with other systems that control neuropathic pain.

Of note, SPMs can act on TRP channels expressed on the surface of sensory neurons, with RvD1 and in particular $\mathrm{RvD}_{2}$ is able to directly inhibit TRP vanilloid subtypes 1, 3, 4 (TRPV1, TRPV3, and TRPV4) and ankyrin 1 (TRPA1) channels, thus preventing in vivo heat-induced pain, inflammatory hypersensitivity, agonist-induced pain and nociception-related excitatory postsynaptic currents (Bang et al., 2010; Park et al., 2011b). Interestingly, also an interaction with opioid receptor has been described. Oehler and colleagues have recently reported that the antinociceptive properties of $\mathrm{RvD}_{1}$-and ChemR23-associated signalling can be abolished by pharmacological blockade of the $\mu$-opioid peptide receptor (MOR) (Oehler et al., 2017). Such an effect did not seem to be related to a direct activation of MOR by SPMs, nor did SPMs induce any release of MOR ligands like $\beta$-endorphin; thus, it was suggested that a cross-talk between MOR and TRP channels signalling was taking place (Oehler et al., 2017). Furthermore, SPMs might alleviate neuropathic pain by acting synergically with other immunomodulating lipids, such as endocannabinoids, in that not only intrathecal administration of $\mathrm{RvD}_{1}$ led to reduced mechanical allodynia and heat hyperalgesia in bone tumour-bearing mice, but also improved the production of $\mathrm{N}$-arachidonoylethanolamine (AEA, also known as anandamide) and 2-arachidonoylglycerol (2-AG) in the spinal cord of these animals (Khasabova et al., 2020). Of note, RvD2 receptor GPR18 is known to engage endocannabinoids, which have been postulated as possible adjuvants in pain treatment (Guerrero-Alba et al., 2019).

Also (neuro)protectins (PDs) have been studied in the past decade with respect to their ability to blunt neuropathic pain. Interestingly, GPR37 has only recently been characterized as receptor of $\mathrm{PD}_{1}$ (Bang et al., 2018), but it was known for its antinociceptive properties ways before the discovery of its proresolving ligand. GPR37 is expressed in macrophages, suggesting that these immune cells play a role in modulating neuroinflammatory pain, as also demonstrated by the presence of GPR37-positive macrophages in mouse samples of spinal cord (Bang et al., 2018, 018). Furthermore, local inflammation upregulates GPR37 in macrophages, and ablation of GPR37 in mice leads to delayed resolution of inflammatory pain (Bang et al., 2018). Interestingly, microglial cells do not express GPR37, suggesting that this receptor might exert its analgesic effects through the secretion of some macrophage-related soluble factors (e.g., IL-10), which in turn blunt peripheral neuroinflammation and reduce nociception in pain-sensing primary sensory neurons (Bang et al., 2018; Chen et al., 2020). Furthermore, previous studies reported a reduction of glial reaction upon local injection of $\mathrm{PD}_{1}$ in mouse models of sciatic nerve transection, suggesting that the latter SPM might act through multiple pathways, yet to be fully understood (Xu et al., 2013). Consistently, GPR $37^{-/-}$mice display enhanced mechanical and cold allodynia, as well as thermal hyperalgesia during bacterial infections, while macrophages primed with GPR37 agonists reduce mechanical allodynia in the same GPR37 ${ }^{-1-}$ mice (Bang et al., 2021). Also $\mathrm{PD}_{1}$ has been demonstrated to counteract neuropathic pain by directly targeting sensory nociception via TRPV1 inhibition in DRG neurons (Park et al., 2011a), and by reversing nerve injuryinduced spinal cord synaptic plasticity in nerve trauma (Xu et al., 2013). Moreover, $\mathrm{PD}_{1}$ is able to induce the expression of brain-derived neurotrophic factor (BDNF) in injured cornea, where it might prevent neuropathic pain by preventing corneal nerves damage (Pham et al., 2017; Pham and Bazan, 2021). Recently, the $\mathrm{PD}_{1}$ synthetic analog 3-oxo- $\mathrm{PD}_{1 \mathrm{n}-3}$ DPA has been reported to exert antinociceptive activity on neuropathic pain associated to streptozocin-induced diabetes in mice (Irina Nesman et al., 2021).

To date, only a few studies have interrogated possible antinociceptive actions of MaRs in models of neuropathic pain. Intrathecal administration of $\mathrm{MaR}_{1}$ inhibits the activation of DRG neurons and abates activation of spinal NF$\mathrm{kB}$ and production of TNF- $\alpha$ and IL- $1 \beta$, resulting in reduced mechanical and thermal hyperalgesia in CFA- and carrageenaninduced pain paradigms, up to 3-5 days after the treatment (Fattori et al., 2019) and in spinal nerve ligation models (Gao et al., 2018). $\mathrm{MaR}_{1}$ also reduces mechanical allodynia in rat models of radicular pain (Wang et al., 2020), as well as chemotaxis of inflammatory cells in the calcitonin gene-related peptide (CGRP)-releasing DRG neurons (Fattori et al., 2019). In line with this, a recent investigation has shown that intraperitoneal administration of $\mathrm{MaR}_{1}$ in a $\mathrm{K} / \mathrm{BxN}$ transfertbased model of arthritic pain led to reduced mechanical hypersensitivity, due to inhibition of macrophage inflammatory chemotaxis in the DRG (Allen et al., 2020). Similarly to other SPMs, therapeutic properties of $\mathrm{MaR}_{1}$ have been linked to its ability to act on vanilloid receptors. Indeed, this lipid showed anti-nociceptive properties in capsaicin-induced spinal and cranial pain, by specifically acting on TRPV1- (but not TRPA1-) induced currents (Serhan et al., 2012; Park, 2015). It seems noteworthy that MaR1 is coupled to $G_{\alpha I}$ proteins, in that pertussis toxin (PTX) reverses its therapeutic activity (Serhan et al., 2012; Park, 2015). This observation has been confirmed when LGR6 was recognized as the G protein-coupled receptor responsible for the effects of $\mathrm{MaR}_{1}$ (Chiang et al., 2019). It should be stressed that LGR6 has not yet been investigated in neuropathic pain, where it might represent a valuable target for future therapeutic strategies.

\section{EPA-Derived SPMs}

E-series resolvins share a number of anti-nociceptive properties with DHA-derived SPMs. $\mathrm{RvE}_{1}$ was firstly described to suppress inflammatory spinal nociception in CFA-, carrageenan- and formalin-induced pain ( $\mathrm{Xu}$ et al., 2010). In these paradigms, pain manifests as a biphasic process, the first phase coming from activation of nociceptive receptors, and the second manifesting as a change in the activity of the spinal neurons following the first phase (Ji et al., 1999). Preemptive administration of $\mathrm{RvE}_{1}$ in formalin-induced pain was only effective in the second phase of pain, suggesting an action of this SPM on central nociceptive signals (Xu et al., 2010). Furthermore, RvEs are also able to modulate pain by interacting with other systems involved in nociceptive signalling. For instance, co-expression of ChemR23 and TRPV1 in DRG neurons has been described, suggesting that 
inhibition of TRPV1 might depend on ChemR23 stimulation by $\mathrm{RvE}_{1}$ (Xu et al., 2010; Jo et al., 2016). Should this interaction be confirmed, SPM receptors and TRP channels might be part of an homeostatic system involved in the genesis of inflammatory neuropathic pain on nociceptive and DRG neurons. Furthermore, RvEs share also with DHA-derived SPMs the ability to modulate signalling of opioids (Oehler et al., 2017), as well as of other lipids, as supported by the observation that $\mathrm{RvE}_{1}$ (like $\mathrm{RvD}_{1}$ ) induces spinal synthesis of endocannabinoids, and together they abate bone cancer-associated pain (Khasabova et al., 2020).

The potential role of ChemR23-dependent signalling in antinociception is also advocated by the finding that chemerin (its firstly identified ligand), or peptide derivatives of this molecule, display analgesic properties by reducing spinal pain (Xu et al., 2010; Doyle et al., 2014), and inhibiting activation of C-fibers (Dickie and Torsney, 2014); Interestingly, $\mathrm{RvE}_{1}$ is the only $\mathrm{SPM}$ as yet known that is able to promote resolution not only by engaging its own receptor-which in turn contributes to the deactivation of the inflammatory wave-but also by acting as a direct antagonist of the $\mathrm{LTB}_{4}$ receptor BLT1. The latter represents a prominent member among pro-inflammatory eicosanoids that participate to inflammation and pain (Inoue and Tsuda, 2018), indicating that resolution of neuropathic pain might indeed be a convoluted process, where SPMs act in synergy, directly or indirectly, with other antinociceptive signals.

\section{CONCLUSION}

Neuropathic pain represents a common and invalidating impairment that undermines the overall quality of life of many patients, for which there is an unmet need of therapeutic treatments especially in the long term. Chronic inflammation represent a very common cause of neuropathic pain, and SPMs are powerful lipid signals that control the resolution of inflammation and prevent many aberrancies of uncontrolled, or prolonged, phlogistic processes including pain. Anti-nociception is exerted by SPMs via three main mechanisms by: i) directly controlling activation of immune cells, their chemotaxis and the release of cytokines; ii) targeting the activation of TRP channels (mostly TRPV1 and TRPA1), for which SPMs represent the first endogenous inhibitors ever described (Park et al., 2011b, 1; Lim et al., 2015); and iii) interacting with opioid signalling.

These observations suggest that SPMs exert a rather pleiotropic activity on the onset of pain, which is achieved by targeting the neuroinflammatory cues that lead to hypersensitivity of nociceptive fibers, and by directly affecting the neuronal processes that lead to pain signal release. Of note, different SPMs seem to drive resolution of inflammatory pain by modulating distinct targets (Fonseca et al., 2017; Valdes et al., 2017; Luo et al., 2019), overall suggesting that these lipids might intervene in different moments, contexts, and possibly types of injuries in order to alleviate pain. One should also consider that, despite the large number of SPMs that have been identified so far, only a handful of SPM receptors have been characterized, and some of them have never been investigated in pain paradigms: one such example is GPR101, which engages the n-3 docosapentaenoic acid (DPA)-derived analogue of RvD5 (Flak et al., 2020). Furthermore, several SPM receptors which have not yet been investigated in pain (see Figure 1C) are nonetheless expressed in neurons, astrocytes and microglia (Tiberi and Chiurchiù, 2021), and might well play a role in the modulation of neuropathic pain.

Oxidative stress is another mechanism that drives inflammation and neuroinflammation, as well as neuropathic pain. Of note, a growing body of evidence has demonstrated the ability of SPMs to blunt the damage caused by excessive production of reactive oxygen and nitrogen species (ROS and RNS, respectively), either by reducing their production or by potentiating anti-oxidative defence systems such as the Kelchlike ECH associated protein 1 (Keap1)/nuclear factor erythroid 2 -related factor 2 (Nrf2) pathway, superoxide dismutase (SOD) and glutathione peroxidase (GSH-PX) (Leuti et al., 2019). Of note, aberrant production of ROS and RNS, coming from deviant and unresolved inflammatory response, not only directly damages sensitive structure that contribute to the transmission of the nociceptive signals (e.g., DRG neurons) but also perpetuates a vicious cycle by activating glial cells, inducing leukocyte recruitment and overall fostering the inflammatory torrent that ultimately leads to neuropathic inflammatory pain. As a result, unbalanced oxidative stress plays a major role in peripheral neuropathies such as those caused by diabetes, alcohol abuse or chemotherapy (Carrasco et al., 2018). Interestingly, TRP channels can be activated by free radicals (Carrasco et al., 2018), suggesting not only that they represent a pivotal element in the genesis of neuropathic pain, but also that SPMs might abate pain by acting on these channels both directly (as described in the present review) and indirectly by interfering with ROS/RNS-mediated damage. To date, however, the direct relationship between pro-resolving lipids, oxidative stress and neuropathic pain has never been directly addressed as a whole, and though strongly suggested by accumulated evidence, it remains a merely speculative mechanism.

It should be noted that microglial cells play a crucial role in mediating spinal pain in several pathologies, and SPMs have consistently been reported to act on the same cells by multiple mechanisms (Tiberi and Chiurchiù, 2021).

Also of relevance, not only SPMs represent very well-tolerated molecules, but some studies also reported their analgesic effect at much lower doses than morphine or COX2 inhibitors (Xu et al., 2010).

It should be noted that SPMs-related dysfunctions have been described in a number of pathologies that feature chronic neuropathic pain as the main or a collateral feature, such as SCI, Parkinson's disease and MS (Prüss et al., 2013; Bisicchia et al., 2018; Krashia et al., 2019; Kooij et al., 2020; Derada Troletti et al., 2021), suggesting that these lipids represent valuable targets for both a better understanding of the pathogenesis of neuropathic pain and the future development of therapeutic strategies that counteract hyperalgic signals by enhancing resolution of inflammation. On a final note, most of the 
investigations published so far were performed by administered SPMs topically, intraperitoneally or intrathecally: additional studies that investigate the efficacy of these lipids through other administration pathways (e.g., oral, sublingual) will be required in order to better evaluate their antinociceptive properties, as well as to formulate new therapeutic strategies; on the other hand, DHA and EPA, as well as SPM precursors are currently commercialized as integrators or nutraceuticals, and a number of studies have reported the beneficial effects of PUFA administration in pain animal models as well as in patients (Ko et al., 2010; Galán-Arriero et al., 2017; Silva et al., 2017).

\section{REFERENCES}

Abdelmoaty, S., Wigerblad, G., Bas, D. B., Codeluppi, S., Fernandez-Zafra, T., ElAwady, E.-S., et al. (2013). Spinal Actions of Lipoxin A4 and 17(R)-resolvin D1 Attenuate Inflammation-Induced Mechanical Hypersensitivity and Spinal TNF Release. PLoS One 8, e75543. doi:10.1371/journal.pone.0075543

Allen, B. L., Montague-Cardoso, K., Simeoli, R., Colas, R. A., Oggero, S., Vilar, B., et al. (2020). Imbalance of Proresolving Lipid Mediators in Persistent Allodynia Dissociated from Signs of Clinical Arthritis. PAIN 161, 2155-2166. doi:10.1097/ j.pain.0000000000001908

Bang, S., Donnelly, C. R., Luo, X., Toro-Moreno, M., Tao, X., Wang, Z., et al. (2021). Activation of GPR37 in Macrophages Confers protection against Infection-Induced Sepsis and Pain-like Behaviour in Mice. Nat. Commun. 12, 1704. doi:10.1038/s41467-021-21940-8

Bang, S., Xie, Y.-K., Zhang, Z.-J., Wang, Z., Xu, Z.-Z., and Ji, R.-R. (2018). GPR37 Regulates Macrophage Phagocytosis and Resolution of Inflammatory Pain. J. Clin. Invest. 128, 3568-3582. doi:10.1172/JCI99888

Bang, S., Yoo, S., Yang, T., Cho, H., Kim, Y., and Hwang, S. (2010). Resolvin D1 Attenuates Activation of Sensory Transient Receptor Potential Channels Leading to Multiple Anti-nociception. Br. J. Pharmacol. 161, 707-720. doi:10.1111/j.1476-5381.2010.00909.x

Baron, R. (2006). Mechanisms of Disease: Neuropathic Pain-A Clinical Perspective. Nat. Rev. Neurol. 2, 95-106. doi:10.1038/ncpneuro0113

Baron, R. (2000). Peripheral Neuropathic Pain: from Mechanisms to Symptoms. The Clin. J. Pain 16, S12-S20. doi:10.1097/00002508-200006001-00004

Bisicchia, E., Sasso, V., Catanzaro, G., Leuti, A., Besharat, Z. M., Chiacchiarini, M., et al. (2018). Resolvin D1 Halts Remote Neuroinflammation and Improves Functional Recovery after Focal Brain Damage via ALX/FPR2 ReceptorRegulated MicroRNAs. Mol. Neurobiol. 55, 6894-6905. doi:10.1007/s12035018-0889-z

Breivik, H., Collett, B., Ventafridda, V., Cohen, R., and Gallacher, D. (2006). Survey of Chronic Pain in Europe: Prevalence, Impact on Daily Life, and Treatment. Eur. J. Pain 10, 287. doi:10.1016/j.ejpain.2005.06.009

Carrasco, C., Naziroğlu, M., Rodríguez, A. B., and Pariente, J. A. (2018). Neuropathic Pain: Delving into the Oxidative Origin and the Possible Implication of Transient Receptor Potential Channels. Front. Physiol. 9, 95. doi:10.3389/fphys.2018.00095

Chen, L., Lv, F., and Pei, L. (2014). Annexin 1: a Glucocorticoid-Inducible Protein that Modulates Inflammatory Pain. Eur J Pain 18, 338-347. doi:10.1002/j.15322149.2013.00373.x

Chen, O., Donnelly, C. R., and Ji, R.-R. (2020). Regulation of Pain by NeuroImmune Interactions between Macrophages and Nociceptor Sensory Neurons. Curr. Opin. Neurobiol. 62, 17-25. doi:10.1016/j.conb.2019.11.006

Chiang, N., Libreros, S., Norris, P. C., de la Rosa, X., and Serhan, C. N. (2019). Maresin 1 Activates LGR6 Receptor Promoting Phagocyte Immunoresolvent Functions. J. Clin. Invest. 129, 5294-5311. doi:10.1172/JCI129448

Chiang, N., and Serhan, C. N. (2017). Structural Elucidation and Physiologic Functions of Specialized Pro-resolving Mediators and Their Receptors. Mol. Aspects Med. 58, 114-129. doi:10.1016/j.mam.2017.03.005

Chiurchiù, V., Leuti, A., Dalli, J., Jacobsson, A., Battistini, L., Maccarrone, M., et al. (2016). Proresolving Lipid Mediators Resolvin D1, Resolvin D2, and Maresin 1

\section{AUTHOR CONTRIBUTIONS}

All authors listed have made a substantial, direct, and intellectual contribution to the work and approved it for publication.

\section{FUNDING}

This investigation was partly supported by the Italian Ministry of Health through the competitive Ricerca Finalizzata 2018 grant (RF-2018-12365391) to MM.

Are Critical in Modulating T Cell Responses. Sci. Transl. Med. 8, 353ra111. doi:10.1126/scitranslmed.aaf7483

Clària, J., and Serhan, C. N. (1995). Aspirin Triggers Previously Undescribed Bioactive Eicosanoids by Human Endothelial Cell-Leukocyte Interactions. Proc. Natl. Acad. Sci. 92, 9475-9479. doi:10.1073/pnas.92.21.9475

Derada Troletti, C., Enzmann, G., Chiurchiù, V., Kamermans, A., Tietz, S. M., Norris, P. C., et al. (2021). Pro-resolving Lipid Mediator Lipoxin A4 Attenuates Neuro-Inflammation by Modulating T Cell Responses and Modifies the Spinal Cord Lipidome. Cel Rep. 35, 109201. doi:10.1016/j.celrep.2021.109201

Dickie, A. C., and Torsney, C. (2014). The Chemerin Receptor 23 Agonist, Chemerin, Attenuates Monosynaptic C-Fibre Input to Lamina I Neurokinin 1 Receptor Expressing Rat Spinal Cord Neurons in Inflammatory Pain. Mol. Pain 10, 1744-8069. doi:10.1186/1744-8069-10-24

Doyle, J. R., Krishnaji, S. T., Zhu, G., Xu, Z.-Z., Heller, D., Ji, R.-R., et al. (2014). Development of a Membrane-Anchored Chemerin Receptor Agonist as a Novel Modulator of Allergic Airway Inflammation and Neuropathic Pain. J. Biol. Chem. 289, 13385-13396. doi:10.1074/jbc.M113.522680

Fattori, V., Pinho-Ribeiro, F. A., Staurengo-Ferrari, L., Borghi, S. M., Rossaneis, A. C., Casagrande, R., et al. (2019). The Specialised Pro-resolving Lipid Mediator Maresin 1 Reduces Inflammatory Pain with a Long-lasting Analgesic Effect. Br. J. Pharmacol. 176, 1728-1744. doi:10.1111/bph.14647

Flak, M. B., Koenis, D. S., Sobrino, A., Smith, J., Pistorius, K., Palmas, F., et al. (2019). GPR101 Mediates the Pro-resolving Actions of RvD5n-3 DPA in Arthritis and Infections. J. Clin. Invest. 130, 359-373. doi:10.1172/JCI131609

Fonseca, F., Orlando, R., Turchetti-Maia, R., and Francischi, J. (2017). Comparative Effects of the $\omega 3$ Polyunsaturated Fatty Acid Derivatives Resolvins E1 and D1 and Protectin DX in Models of Inflammation and Pain. J Inflamm Res 10, 119-133. doi:10.2147/JIR.S142424

Galán-Arriero, I., Serrano-Muñoz, D., Gómez-Soriano, J., Goicoechea, C., Taylor, J., Velasco, A., et al. (2017). The Role of Omega-3 and Omega-9 Fatty Acids for the Treatment of Neuropathic Pain after Neurotrauma. Biochim. Biophys. Acta (Bba) - Biomembranes 1859, 1629-1635. doi:10.1016/j.bbamem.2017.05.003

Gao, J., Tang, C., Tai, L. W., Ouyang, Y., Li, N., Hu, Z., et al. (2018). Pro-resolving Mediator Maresin 1 Ameliorates Pain Hypersensitivity in a Rat Spinal Nerve Ligation Model of Neuropathic Pain. J Pain Res 11, 1511-1519. doi:10.2147/ JPR.S160779

Guerrero-Alba, R., Barragán-Iglesias, P., González-Hernández, A., ValdezMoráles, E. E., Granados-Soto, V., Condés-Lara, M., et al. (2019). Some Prospective Alternatives for Treating Pain: The Endocannabinoid System and its Putative Receptors GPR18 and GPR55. Front. Pharmacol. 9. doi:10.3389/fphar.2018.01496

Han, Y.-H., Shin, K.-O., Kim, J.-Y., Khadka, D. B., Kim, H.-J., Lee, Y.-M., et al. (2019). A Maresin 1/RORa/12-Lipoxygenase Autoregulatory Circuit Prevents Inflammation and Progression of Nonalcoholic Steatohepatitis. J. Clin. Invest. 129, 1684-1698. doi:10.1172/JCI124219

Hu, S., Mao-Ying, Q.-L., Wang, J., Wang, Z.-F., Mi, W.-L., Wang, X.-W., et al. (2012). Lipoxins and Aspirin-Triggered Lipoxin Alleviate Bone Cancer Pain in Association with Suppressing Expression of Spinal Proinflammatory Cytokines. J. Neuroinflammation 9, 278. doi:10.1186/1742-2094-9-278

Huang, J., Burston, J. J., Li, L., Ashraf, S., Mapp, P. I., Bennett, A. J., et al. (2017). Targeting the D Series Resolvin Receptor System for the Treatment of Osteoarthritis Pain. Arthritis Rheumatol. 69, 996-1008. doi:10.1002/art.40001 
Huang, L., Wang, C.-F., Serhan, C. N., and Strichartz, G. (2011). Enduring Prevention and Transient Reduction of Postoperative Pain by Intrathecal Resolvin D1. Pain 152, 557-565. doi:10.1016/j.pain.2010.11.021

Inoue, K., and Tsuda, M. (2018). Microglia in Neuropathic Pain: Cellular and Molecular Mechanisms and Therapeutic Potential. Nat. Rev. Neurosci. 19, 138-152. doi:10.1038/nrn.2018.2

Jang, Y., Kim, M., and Hwang, S. W. (2020). Molecular Mechanisms Underlying the Actions of Arachidonic Acid-Derived Prostaglandins on Peripheral Nociception. J. Neuroinflammation 17, 30. doi:10.1186/s12974-020-1703-1

Jardín, I., López, J. J., Diez, R., Sánchez-Collado, J., Cantonero, C., Albarrán, L., et al. (2017). TRPs in Pain Sensation. Front. Physiol. 8. doi:10.3389/ fphys.2017.00392

Ji, R.-R., Baba, H., Brenner, G. J., and Woolf, C. J. (1999). Nociceptive-specific Activation of ERK in Spinal Neurons Contributes to Pain Hypersensitivity. Nat. Neurosci. 2, 1114-1119. doi:10.1038/16040

Jo, Y. Y., Lee, J. Y., and Park, C.-K. (2016). Resolvin E1 Inhibits Substance P-Induced Potentiation of TRPV1 in Primary Sensory Neurons. Mediators Inflamm. 2016, e5259321. doi:10.1155/2016/5259321

Khasabova, I. A., Golovko, M. Y., Golovko, S. A., Simone, D. A., and Khasabov, S. G. (2020). Intrathecal Administration of Resolvin D1 and E1 Decreases Hyperalgesia in Mice with Bone Cancer Pain: Involvement of Endocannabinoid Signaling. Prostaglandins \& Other Lipid Mediators 151, 106479. doi:10.1016/j.prostaglandins.2020.106479

Kidd, B. L., and Urban, L. A. (2001). Mechanisms of Inflammatory Pain. Br. J. Anaesth. 87, 3-11. doi:10.1093/bja/87.1.3

Ko, G. D., Nowacki, N. B., Arseneau, L., Eitel, M., and Hum, A. (2010). Omega-3 Fatty Acids for Neuropathic Pain. Clin. J. Pain 26, 168-172. doi:10.1097/ AJP.0b013e3181bb8533

Kooij, G., Troletti, C. D., Leuti, A., Norris, P. C., Riley, I., Albanese, M., et al. (2020). Specialized Pro-resolving Lipid Mediators Are Differentially Altered in Peripheral Blood of Patients with Multiple Sclerosis and Attenuate Monocyte and Blood-Brain Barrier Dysfunction. Haematologica 105, 2056-2070. doi:10.3324/haematol.2019.219519

Krashia, P., Cordella, A., Nobili, A., La Barbera, L., Federici, M., Leuti, A., et al. (2019). Blunting Neuroinflammation with Resolvin D1 Prevents Early Pathology in a Rat Model of Parkinson's Disease. Nat. Commun. 10, 3945. doi:10.1038/s41467-019-11928-w

Leuti, A., Fazio, D., Fava, M., Piccoli, A., Oddi, S., and Maccarrone, M. (2020). Bioactive Lipids, Inflammation and Chronic Diseases. Adv. Drug Deliv. Rev. 159, 133-169. doi:10.1016/j.addr.2020.06.028

Leuti, A., Maccarrone, M., and Chiurchiù, V. (2019). Proresolving Lipid Mediators: Endogenous Modulators of Oxidative Stress. Oxidative Med. Cell Longevity 2019, 1-12. doi:10.1155/2019/81072652019

Li, Q., Tian, Y., Wang, Z.-F., Liu, S.-B., Mi, W.-L., Ma, H.-J., et al. (2013). Involvement of the Spinal NALP1 Inflammasome in Neuropathic Pain and Aspirin-Triggered-15-Epi-Lipoxin A4 Induced Analgesia. Neuroscience 254, 230-240. doi:10.1016/j.neuroscience.2013.09.028

Lim, J. Y., Park, C.-K., and Hwang, S. W. (2015). Biological Roles of Resolvins and Related Substances in the Resolution of Pain. Biomed. Res. Int. 2015, 1-14. doi:10.1155/2015/830930

Lima-Garcia, J., Dutra, R., da Silva, K., Motta, E., Campos, M., and Calixto, J. (2011). The Precursor of Resolvin D Series and Aspirin-Triggered Resolvin D1 Display Anti-hyperalgesic Properties in Adjuvant-Induced Arthritis in Rats. Br. J. Pharmacol. 164, 278-293. doi:10.1111/j.1476-5381.2011.01345.x

Liu, Z.-h., Miao, G.-s., Wang, J.-n., Yang, C.-x., Fu, Z.-j., and Sun, T. (2016). Resolvin D1 Inhibits Mechanical Hypersensitivity in Sciatica by Modulating the Expression of Nuclear Factor-Kb, Phospho-Extracellular Signal-Regulated Kinase, and Pro- and Antiinflammatory Cytokines in the Spinal Cord and Dorsal Root Ganglion. Anesthesiology 124, 934-944. doi:10.1097/ ALN.0000000000001010

Luo, X., Gu, Y., Tao, X., Serhan, C. N., and Ji, R.-R. (2019). Resolvin D5 Inhibits Neuropathic and Inflammatory Pain in Male but Not Female Mice: Distinct Actions of D-Series Resolvins in Chemotherapy-Induced Peripheral Neuropathy. Front. Pharmacol. 10, 745. doi:10.3389/fphar.2019.00745

Martini, A. C., Berta, T., Forner, S., Chen, G., Bento, A. F., Ji, R.-R., et al. (2016). Lipoxin A4 Inhibits Microglial Activation and Reduces Neuroinflammation and Neuropathic Pain after Spinal Cord Hemisection. J. Neuroinflammation 13, 75. doi:10.1186/s12974-016-0540-8
Meesawatsom, P., Burston, J., Hathway, G., Bennett, A., and Chapman, V. (2016). Inhibitory Effects of Aspirin-Triggered Resolvin D1 on Spinal Nociceptive Processing in Rat Pain Models. J. Neuroinflammation 13, 233. doi:10.1186/ s12974-016-0676-6

Miao, G.-S., Liu, Z.-H., Wei, S.-X., Luo, J.-G., Fu, Z.-J., and Sun, T. (2015). Lipoxin A4 Attenuates Radicular Pain Possibly by Inhibiting Spinal ERK, JNK and NF-kB/p65 and Cytokine Signals, but Not P38, in a Rat Model of Non-compressive Lumbar Disc Herniation. Neuroscience 300, 10-18. doi:10.1016/j.neuroscience.2015.04.060

Nesman, J. I., Chen, O., Luo, X., Ji, R.-R., Serhan, C. N. C., and Hansen, T. V. (2021). A New Synthetic Protectin D1 Analog 3-Oxa-PD1n-3 DPA Reduces Neuropathic Pain and Chronic Itch in Mice. Org. Biomol. Chem. 19, 2744-2752. doi:10.1039/D0OB02136A

Oehler, B., Mohammadi, M., Perpina Viciano, C., Hackel, D., Hoffmann, C., Brack, A., et al. (2017). Peripheral Interaction of Resolvin D1 and E1 with Opioid Receptor Antagonists for Antinociception in Inflammatory Pain in Rats. Front. Mol. Neurosci. 10. doi:10.3389/fnmol.2017.00242

Oh, S. F., Dona, M., Fredman, G., Krishnamoorthy, S., Irimia, D., and Serhan, C. N. (2012). Resolvin E2 Formation and Impact in Inflammation Resolution. J.I. 188, 4527-4534. doi:10.4049/jimmunol.1103652

Park, C.-K., Lü, N., Xu, Z.-Z., Liu, T., Serhan, C. N., and Ji, R.-R. (2011a). Resolving TRPV1- and TNF- -Mediated Spinal Cord Synaptic Plasticity and Inflammatory Pain with Neuroprotectin D1. J. Neurosci. 31, 15072-15085. doi:10.1523/JNEUROSCI.2443-11.2011

Park, C.-K. (2015). Maresin 1 Inhibits TRPV1 in Temporomandibular JointRelated Trigeminal Nociceptive Neurons and TMJ Inflammation-Induced Synaptic Plasticity in the Trigeminal Nucleus. Mediators Inflamm. 2015, 1-9. doi:10.1155/2015/275126

Park, C.-K., Xu, Z.-Z., Liu, T., Lü, N., Serhan, C. N., and Ji, R.-R. (2011b). Resolvin D2 Is a Potent Endogenous Inhibitor for Transient Receptor Potential Subtype V1/A1, Inflammatory Pain, and Spinal Cord Synaptic Plasticity in Mice: Distinct Roles of Resolvin D1, D2, and E1. J. Neurosci. 31, 18433-18438. doi:10.1523/JNEUROSCI.4192-11.2011

Pham, T. L., and Bazan, H. E. P. (2021). Docosanoid Signaling Modulates Corneal Nerve Regeneration: Effect on Tear Secretion, Wound Healing, and Neuropathic Pain. J. Lipid Res. 62, 100033. doi:10.1194/jlr.TR120000954

Pham, T. L., He, J., Kakazu, A. H., Jun, B., Bazan, N. G., and Bazan, H. E. P. (2017). Defining a Mechanistic Link between Pigment Epithelium-Derived Factor, Docosahexaenoic Acid, and Corneal Nerve Regeneration. J. Biol. Chem. 292, 18486-18499. doi:10.1074/jbc.M117.801472

Prüss, H., Rosche, B., Sullivan, A. B., Brommer, B., Wengert, O., Gronert, K., et al. (2013). Proresolution Lipid Mediators in Multiple Sclerosis - Differential, Disease Severity-dependent Synthesis - a Clinical Pilot Trial. PLoS One 8, e55859. doi:10.1371/journal.pone.0055859

Quan-Xin, F., Fan, F., Xiang-Ying, F., Shu-Jun, L., Shi-Qi, W., Zhao-Xu, L., et al. (2012). Resolvin D1 Reverses Chronic Pancreatitis-Induced Mechanical Allodynia, Phosphorylation of NMDA Receptors, and Cytokines Expression in the Thoracic Spinal Dorsal Horn. BMC Gastroenterol. 12, 148. doi:10.1186/1471-230X-12-148

Romano, M., Cianci, E., Simiele, F., and Recchiuti, A. (2015). Lipoxins and AspirinTriggered Lipoxins in Resolution of Inflammation. Eur. J. Pharmacol. 760, 49-63. doi:10.1016/j.ejphar.2015.03.083

Saito, T., Hasegawa-Moriyama, M., Kurimoto, T., Yamada, T., Inada, E., and Kanmura, Y. (2015). Resolution of Inflammation by Resolvin D1 Is Essential for Peroxisome Proliferator-Activated Receptor- $\gamma$-Mediated Analgesia during Postincisional Pain Development in Type 2 Diabetes. Anesthesiology 123, 1420-1434. doi:10.1097/ALN.0000000000000892

Serhan, C. N., Dalli, J., Karamnov, S., Choi, A., Park, C. K., Xu, Z. Z., et al. (2012). Macrophage Proresolving Mediator Maresin 1 Stimulates Tissue Regeneration and Controls Pain. FASEB j. 26, 1755-1765. doi:10.1096/fj.11-201442

Serhan, C. N., Fierro, I. M., Chiang, N., and Pouliot, M. (2001). Cutting Edge: Nociceptin Stimulates Neutrophil Chemotaxis and Recruitment: Inhibition by Aspirin-Triggered-15-Epi-Lipoxin A4. J. Immunol. 166, 3650-3654. doi:10.4049/jimmunol.166.6.3650

Serhan, C. N., Hamberg, M., Samuelsson, B., Morris, J., and Wishka, D. G. (1986). On the Stereochemistry and Biosynthesis of Lipoxin B. Proc. Natl. Acad. Sci. 83, 1983-1987. doi:10.1073/pnas.83.7.1983

Serhan, C. N., and Levy, B. D. (2018). Resolvins in Inflammation: Emergence of the Pro-resolving Superfamily of Mediators. J. Clin. Invest. 128, 2657-2669. doi:10.1172/JCI97943 
Silva, R. V., Oliveira, J. T., Santos, B. L. R., Dias, F. C., Martinez, A. M. B., Lima, C. K. F., et al. (2017). Long-Chain Omega-3 Fatty Acids Supplementation Accelerates Nerve Regeneration and Prevents Neuropathic Pain Behavior in Mice. Front. Pharmacol. 8, 723. doi:10.3389/fphar.2017.00723

Sun, T., Yu, E., Yu, L., Luo, J., Li, H., and Fu, Z. (2012). LipoxinA4 Induced Antinociception and Decreased Expression of NF-Kb and Pro-inflammatory Cytokines after Chronic Dorsal Root Ganglia Compression in Rats. Ejp 16, 18-27. doi:10.1016/j.ejpain.2011.05.005

Svensson, C. I., Zattoni, M., and Serhan, C. N. (2007). Lipoxins and AspirinTriggered Lipoxin Inhibit Inflammatory Pain Processing. J. Exp. Med. 204, 245-252. doi:10.1084/jem.20061826

Tian, Y., Liu, M., Mao-Ying, Q.-L., Liu, H., Wang, Z.-F., Zhang, M.-T., et al. (2015). Early Single Aspirin-Triggered Lipoxin Blocked Morphine Anti-nociception Tolerance through Inhibiting NALP1 Inflammasome: Involvement of PI3k/Akt Signaling Pathway. Brain Behav. Immun. 50, 63-77. doi:10.1016/ j.bbi.2015.06.016

Tiberi, M., and Chiurchiù, V. (2021). Specialized Pro-resolving Lipid Mediators and Glial Cells: Emerging Candidates for Brain Homeostasis and Repair. Front. Cel. Neurosci. 15, 673549. doi:10.3389/fncel.2021.673549

Valdes, A. M., Ravipati, S., Menni, C., Abhishek, A., Metrustry, S., Harris, J., et al. (2017). Association of the Resolvin Precursor 17-HDHA, but Not D- or ESeries Resolvins, with Heat Pain Sensitivity and Osteoarthritis Pain in Humans. Sci. Rep. 7, 10748. doi:10.1038/s41598-017-09516-3

Wang, J. C.-F., and Strichartz, G. R. (2017). Prevention of Chronic PostThoracotomy Pain in Rats by Intrathecal Resolvin D1 and D2: Effectiveness of Perioperative and Delayed Drug Delivery. The J. Pain 18, 535-545. doi:10.1016/j.jpain.2016.12.012

Wang, Y.-H., Li, Y., Wang, J.-N., Zhao, Q.-X., Jin, J., Wen, S., et al. (2020). Maresin 1 Attenuates Radicular Pain through the Inhibition of NLRP3 InflammasomeInduced Pyroptosis via NF-Kb Signaling. Front. Neurosci. 14, 831. doi:10.3389/ fnins.2020.00831
Wang, Z.-F., Li, Q., Liu, S.-B., Mi, W.-L., Hu, S., Zhao, J., et al. (2014). Aspirintriggered Lipoxin A4 Attenuates Mechanical Allodynia in Association with Inhibiting Spinal JAK2/STAT3 Signaling in Neuropathic Pain in Rats. Neuroscience 273, 65-78. doi:10.1016/j.neuroscience.2014.04.052

Xu, Z.-Z., Liu, X.-J., Berta, T., Park, C.-K., Lü, N., Serhan, C. N., et al. (2013). Neuroprotectin/protectin D1 Protects against Neuropathic Pain in Mice after Nerve Trauma. Ann. Neurol. 74, 490-495. doi:10.1002/ana.23928

Xu, Z.-Z., Zhang, L., Liu, T., Park, J. Y., Berta, T., Yang, R., et al. (2010). Resolvins RvE1 and RvD1 Attenuate Inflammatory Pain via central and Peripheral Actions. Nat. Med. 16, 592-597. doi:10.1038/nm.2123

Zhang, L., Terrando, N., Xu, Z.-Z., Bang, S., Jordt, S.-E., Maixner, W., et al. (2018). Distinct Analgesic Actions of DHA and DHA-Derived Specialized Proresolving Mediators on Post-operative Pain after Bone Fracture in Mice. Front. Pharmacol. 9, 412. doi:10.3389/fphar.2018.00412

Conflict of Interest: The authors declare that the research was conducted in the absence of any commercial or financial relationships that could be construed as a potential conflict of interest.

Publisher's Note: All claims expressed in this article are solely those of the authors and do not necessarily represent those of their affiliated organizations, or those of the publisher, the editors and the reviewers. Any product that may be evaluated in this article, or claim that may be made by its manufacturer, is not guaranteed or endorsed by the publisher.

Copyright (c) 2021 Leuti, Fava, Pellegrini and Maccarrone. This is an open-access article distributed under the terms of the Creative Commons Attribution License (CC $B Y)$. The use, distribution or reproduction in other forums is permitted, provided the original author(s) and the copyright owner(s) are credited and that the original publication in this journal is cited, in accordance with accepted academic practice. No use, distribution or reproduction is permitted which does not comply with these terms. 